\title{
The Modified Gravitation-based Algorithm for Community Detecting in Dynamic Social Networks
}

\author{
Mingxue Liao* \\ Institute of Automation Chinese \\ Academy of Sciences, China \\ mingxue.liao@ia.ac.cn
}

\author{
Yuanyuan Liang \\ Institute of Automation Chinese \\ Academy of Sciences, China \\ yuanyuan.liang@ia.ac.cn
}

\author{
Qinying Li \\ Institute of Automation Chinese \\ Academy of Sciences, China \\ qinying.li@ia.ac.cn
}

\begin{abstract}
With in-depth research on the complex network, people have a deeper understanding of the physical significance and mathematical characteristics of social networks. Communities are clusters of closely connected nodes within a social network. Community detection is an important task to understand the characteristics of social networks. The gravitation-based algorithm (GBA) is proposed in our previous work to simulate the process of community evolution based on Newton's law of universal gravitation. Based on GBA, we present a modified gravitation-based algorithm (MGBA) in this paper. MGBA uses the degree of nodes in the network to represent the mass of nodes, redefines the distance parameter in the law of gravity according to the close structural characteristics of the community, and introduces the concepts of the parent node and the center node. MGBA includes the MAGB algorithm and the MAFMG algorithm. MAGB is used to detect the community structure and find the center community. MAFMG algorithm is used to find the max gravity of the community. On this basis of MGBA, we present a dynamic modified gravitation-based algorithm (DMGBA) which is based on incremental clustering. After using MGBA to detect the community structure in static networks. If edges are added or removed, DMGBA will be used to redivide the dynamical communities. For adding a new edge, add the neighbor nodes of two related nodes, modify the quality of each node, and then put these nodes into a collection. For deleting old edges, delete the neighbor nodes of two related nodes, and modify the mass of each node. Change the central node of related nodes to itself, and put them into the collection if they are still in the network. The experimental results show that MGBA algorithm achieves $1 \%$ higher F1-score in less time than GBA in static large-scale social networks, DMGBA can get good partitions of communities in much less time than comparison methods.
\end{abstract}

\section{CCS CONCEPTS}

- Human-centered computing; - Collaborative and social computing; $\bullet$ Collaborative and social computing design and evaluation methods; • Social network analysis;

*Corresponding author: mingxue.liao@ia.ac.cn

CC.
This work is licensed under a Creative Commons Attribution International
4.0 License.
ACAI'21, December 22-24, 2021, Sanya, China
(c) 2021 Copyright held by the owner/author(s). Publication rights licensed to ACM.
ACM ISBN $978-1-4503-8505-3 / 21 / 12$.
https://doi.org/10.1145/3508546.3508583

\section{KEYWORDS}

Social Networks, Community Detection, MGBA, DMGBA

\section{ACM Reference Format:}

Mingxue Liao, Yuanyuan Liang, and Qinying Li. 2021. The Modified Gravitation-based Algorithm for Community Detecting in Dynamic Social Networks. In 2021 4th International Conference on Algorithms, Computing and Artificial Intelligence (ACAI'21), December 22-24, 2021, Sanya, China. ACM, New York, NY, USA, 6 pages. https://doi.org/10.1145/3508546.3508583

\section{INTRODUCTION}

Communities are clusters of closely connected nodes within a social network. Many algorithms are used to detect the community structure, with different grouping criteria depending on the practical method [1] or the basic concept of community [2]. Blondel presents the Louvain algorithm [3] to detect communities in large-scale networks. The smart local moving (SLM) algorithm [4] is based on the local movement heuristic algorithm which can identify community structures with higher modularity than the Louvain algorithm. Cai refers to a community detection algorithm [5] based on spectral clustering and modularity function, which can efficiently obtain high-quality community structures in large networks. The GBA which is proposed in our previous work simulates the process of community evolution based on Newton's law of universal gravitation. Most of the above researches only detect community structures of static networks, and those algorithms don't perform well in large-scale networks.

Due to communities in the real world continuing to evolve as dynamic networks change, many current dynamic community detection algorithms are presented to study the trend of community change over time [6]. For instance, Hopcroft analyzes the network snapshots from 1990 to 2001 provided by the NEC CiteSeer database [7], using a hierarchical clustering algorithm to detect the communities in snapshots. Besides, some algorithms define community detection in dynamic networks as a multi-objective optimization problem to improve the effectiveness and accuracy [8-10]. The classic genetic operators in several evolutionary clustering methods limit their effectiveness. To overcome this problem, Liu proposes a new migration operator that works in collaboration with classical genetic operators to improve the discovery of evolutionary community structure [11]. Meng puts forward a dynamic Louvain algorithm, which combines historical information and incremental information to detect communities in dynamic networks based on modular optimization [12]. Zhao makes a comprehensive analysis of the network before and after the change, and puts forward four incremental events and corresponding update strategies to detect the communities in the dynamically changing social network [13]. 
$\mathrm{Xu}$ presents a two-stage method to track the evolution of communities in dynamic networks. The first phase estimates the degree of error accumulation of incremental clustering for each snapshot, and the second phase identifies critical evolutionary events [14].

For a dynamic network, they adjust the communities via the different variations of the core node chains to simulate the evolutionary processes of the communities based on the results of network snapshots. Like static algorithms, the running time of these dynamic algorithms may be further reduced. Besides, since most of the dynamic algorithms are based on the search results of the selected static algorithms and incremental changes, these algorithms cannot be applied to large-scale networks with the limits of static algorithms. In summary, there have been in-depth and extensive researches on community detection algorithms, but most of the algorithms have not performed well in the application of large-scale networks.

In this paper, we present a modified gravitation-based algorithm (MGBA) based on our previous work gravity-based algorithm (GBA). The GBA uses Newton's law of universal gravitation to simulate the process of community evolution in large-scale networks, so MGBA can detect communities in static large-scale networks. On the basis of MGBA, we put forward the dynamic gravitation-based algorithm (DMGBA). DMGBA can detect communities in dynamic networks based on incremental changes. The algorithm [15] is also based on gravitational relationships, but our algorithms are different in the evolutionary process of the community and the parameter definition. Besides, our algorithms conduct experiments in large-scale networks with tens of millions of nodes while the algorithm [15] doesn't.

This article is organized as follows. In Section 2, we introduce our previous work GBA algorithm. In Section 3, the MGBA and DMGBA are presented and illustrated. Experimental results are given in Section 4. Section 5 is the conclusion and future work.

\section{RELATED WORK}

GBA is proposed in our previous work. GBA detects community by using the thought of Galaxy Evolution. Each community can be seen as a galaxy which can be nested. Discovering the community is similar to the natural evolution of galaxies. In social networks, the degree of a node can partly reflect the local influence of the node. Node mass is calculated by degree, community mass is the mass of all nodes $m_{C}=\sum\left(m_{n}\right)$, the center node mass is the community mass. By using hop to represent distance, the gravitation can be calculated as:

$$
G=g \frac{m_{1} * m_{2}}{h o p^{2}}
$$

Where $G$ is the gravitation between two communities, $g$ is the universal gravitation constant and we set it to $1, m_{1}$ is the mass of community $1, m_{2}$ is the mass of community 2 , hop represents the number of records that have arrived during the search process. And hop can be calculated by

$$
\begin{gathered}
\text { Hop }=\sum_{i=1}^{M A X_{-} H O P}\left(\frac{b_{i}}{w_{i}}\right)^{2} \\
m=\alpha d+\beta p
\end{gathered}
$$

$w_{1}$ represents the weight of the first hop, $w_{2}$ represents the weight of the second hop and so on. For simplicity, their values are set to 1. And $m$ is the mass of a node, $d$ is the degree of a node, $p$ is the property of a node.

\section{METHODOLOGY}

\subsection{MGBA}

On the basis of the GBA, we present MGBA to simulate the process of community evolution. MGBA can detect communities in static large-scale networks. In MGBA, we present the concept of the parent node. When a vertex community is allocated to the community with the largest gravity, the central node of this community is defined as the parent node of the vertex community. Once assigned, the parent node of a community will not be changed anymore, while the central node of a community will change with the operation of the gravity model changing. Every vertex community's center node is the node itself at the beginning, then it will change as the central node of the parent node changes. Besides, we use dis to represent the distance of communities to replace the hops. According to formula (1), the closer the distance between objects is, the greater gravity is. Therefore, dis is defined as the inverse proportional function of the number of connected nodes belonging to the same central community. It conforms to the basic idea of the gravity calculation formula and the goal of community detection. And dis can be calculated by

$$
\text { dis }=\frac{1}{C_{i}}
$$

$C_{i}$ represents the number of connected nodes of a node attached to the $i$ community. Then the gravitation can be calculated as:

$$
G=g \frac{m_{1} * m_{2}}{d i s^{2}}
$$

The MGBA includes the modified adaptive gravity-based (MAGB) algorithm and the modified adaptive find_max gravity (MAFMG) algorithm just like the GBA. The ALGORITHM 1 shows the steps of the MAGB algorithm. It is used to find the max gravity of the community for each vertex community by calling MAFMG algorithm and update the substructure when center nodes change. The ALGORITHM 2 shows the steps of the MAFMG algorithm. It finds the central node for the vertex community by using formula (5).

\subsection{DMGBA}

On the basis of the MGBA, we put forward the DMGBA. DMGBA can detect communities in dynamic networks based on incremental changes. The nodes and edges of a dynamic network will change with time, and the communities will evolve with changes. In the previous research, a dynamic network $G$ is a sequence of network snapshots, and it can be presented as $G_{1}, G_{2}, \ldots, G_{t}$ where $G_{t}$ is the temporal graph at time $t$. There are four basic events between $G_{t}$ and $G_{t+1}$ : (1) the leaving of the original nodes; (2) the leaving of the original edges; (3) the addition of new edges; (4) the addition of new nodes. But if a node leaves the network, all of the relevant edges also leave. If a node joins, the relevant edges also join. Therefore, we reduced the basic events to two: (1) the leaving of the original edges; (2) the addition of new edges. Then adjust the community structure obtained by using the MGBA. 

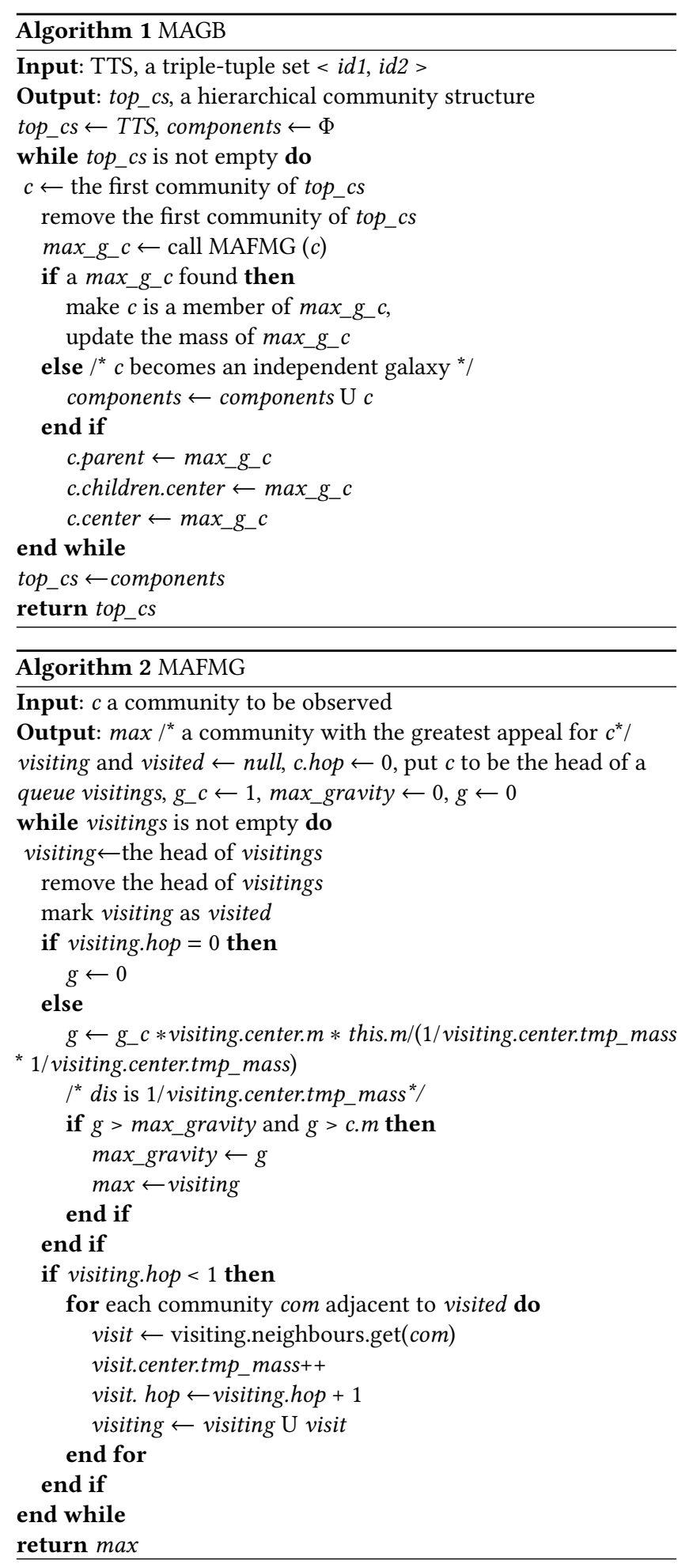

For the addition of new edges, add the neighbor node of the two related nodes and modify the mass of each node, then put these nodes into a collection. For the leaving of the original edges, delete the neighbor node of the two related nodes and modify the mass of each node. Change the central node of the relevant node to itself and put these nodes into the collection if they are still in the network.

The DMGBA includes the dynamic modified adaptive gravitybased (DMAGB) algorithm and the dynamic modified adaptive find_max gravity (DMAFMG) algorithm. The ALGORITHM 3 shows the steps of the DMAGB algorithm. It is used to find the max gravity of the community for each node in the collection mentioned above by calling DMAFMG algorithm and update the substructure when center nodes change. The steps of the DMAFMG algorithm are the same as the MAFMG algorithm.

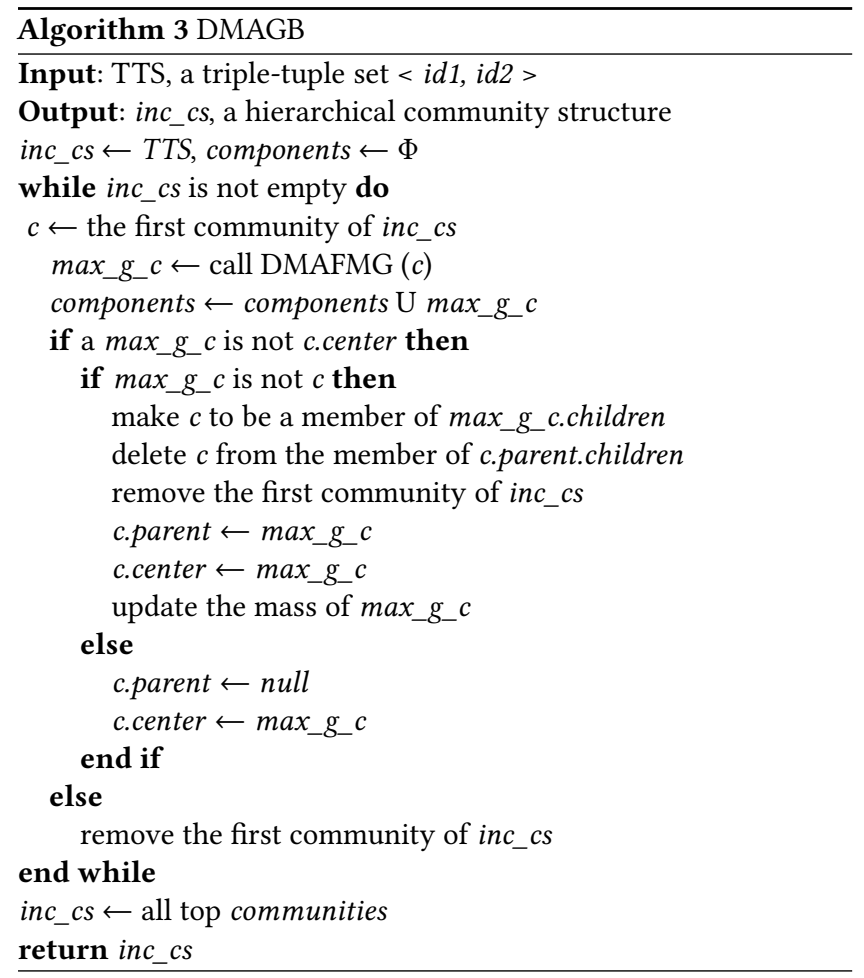

\section{EXPERIMENT RESULTS}

\subsection{Experiments in static networks}

4.1.1 Experimental Environment and Program. We compare the computational time of these algorithms by running a series of experiments on Ubuntu 16.04 LTS with Intel @ Xeon (R) CPU E52699 v4@2.20GHz 52 and 250GB RAM. To verify the accuracy of the experiment results, we choose F1-score to evaluate the performance of the MGBA.

4.1.2 Experiments Datasets. We download five networks (comLiveJournal [16], com-DBLP [16], com-YouTube [17], com-Orkut [18], com-Amazon [19]) from the Stanford Large Network Datasets Collection (http://snap.stanford.edu/data/) to evaluate the performance of the MGBA and GBA. The com-LiveJournal, com-Orkut 
Table 1: Experiments Datasets

\begin{tabular}{lll}
\hline Datasets & Edges & Nodes \\
LiveJournal & $34,681,189$ & $3,997,962$ \\
Orkut & $117,185,083$ & $3,072,441$ \\
YouTube & $2,987,624$ & $1,134,890$ \\
DBLP & $1,049,866$ & 317,080 \\
Amazon & 925872 & 334863 \\
\hline
\end{tabular}

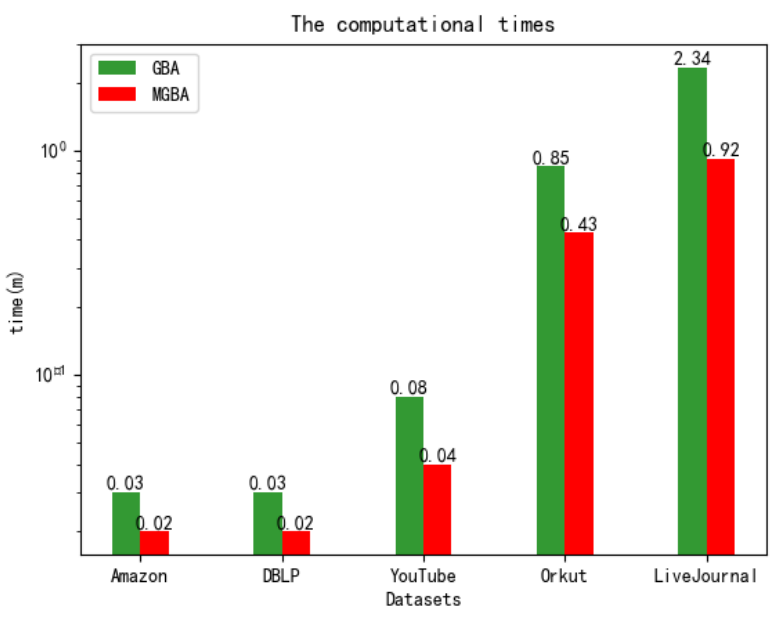

Figure 1: The computational times of GBA and MGBA.

and com-YouTube are online social networks. The com-DBLP is a collaboration network and the com-Amazon is a product network.

4.1.3 Experimental Results - Efficiency. We compare the computational time of GBA and MGBA. Fig.1 shows that MGBA runs faster than GBA on all datasets. This is because MGBA puts forward the concept of parent node. When selecting the largest gravitational community for each node, MGBA only needs to investigate the neighbor nodes to adjust the hierarchical nested community structure, which reduces the running time of the algorithm.

4.1.4 Experimental Results - Accuracy. We choose a F1-score to compare the accuracy of the experiment results. F1-score is a measure of the similarity between the best-matching detected community and each ground-truth community [20].

$$
F 1=\frac{1}{2}\left(\frac{1}{\left|C^{*}\right|} \sum_{i} \max _{j} F 1\left(C_{i}, \widehat{C}_{j}\right)+\frac{1}{|\hat{C}|} \sum_{i} \max _{j} F 1\left(C_{j}, \widehat{C}_{i}\right)\right)
$$

We generate a set of relatively small subnetworks with community structures to allow for comparison [21]. We created 100 different subnetworks for these five datasets.

Table 2 shows the relative improvement of MGBA over the GBA. For instance, 0.02 for F1-score on YouTube means that MGBA achieves $2 \%$ higher F1-score than GBA. In conclusion, the accuracy of MGBA performs best in all datasets. On average, MGBA achieves $1.4 \%$ higher F1-score by less time than GBA. The experimental results show that MGBA is more effective than GBA.

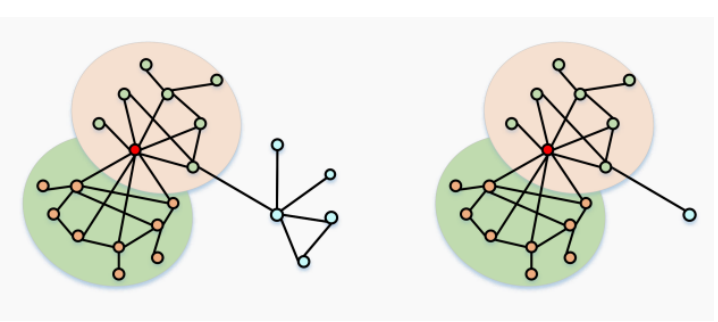

Figure 2: The accuracy of GBA and MGBA.

Table 2: Relative improvement in F1-score of MGBA over GBA in experimental datasets

\begin{tabular}{cc}
\hline Datasets & F1-score \\
Amazon & 0.00 \\
DBLP & 0.00 \\
YouTube & 0.02 \\
LiveJournal & 0.01 \\
Orkut & 0.04 \\
Average & $\mathbf{0 . 0 1 4}$ \\
\hline
\end{tabular}

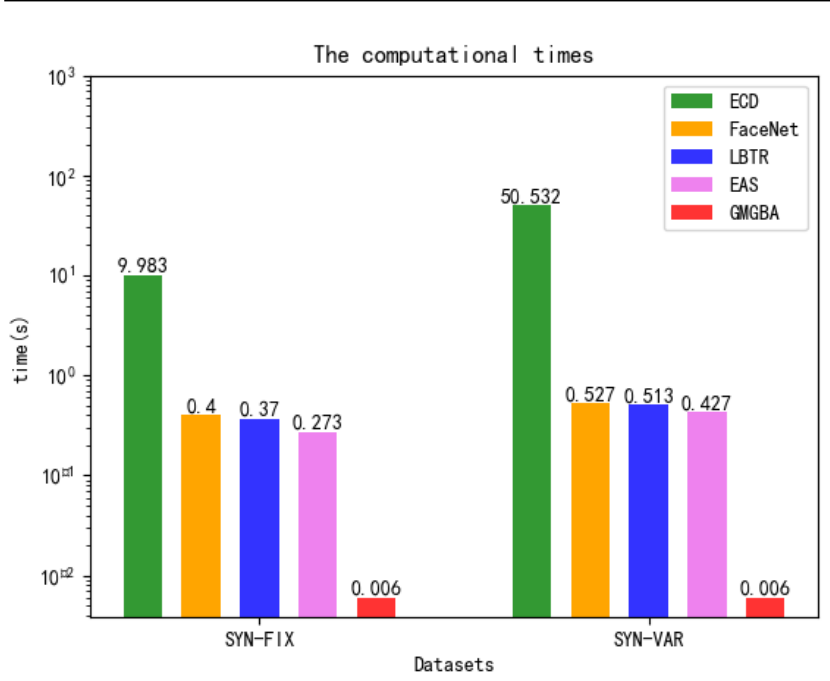

Figure 3: The computational times of DMGBA and compared methods. 

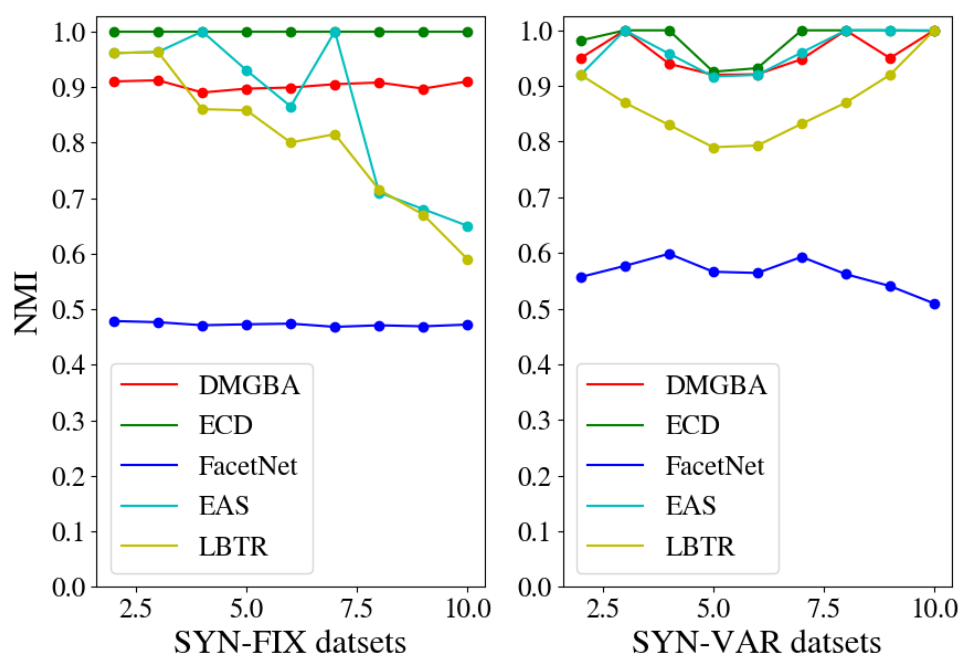

Figure 4: The experimental accuracy of DMGBA and compared methods.

\subsection{Experiments in dynamic networks}

4.2.1 Experimental Environment and Program. We evaluate the algorithm performance of the DMGBA and baseline algorithms on a PC with $2.30 \mathrm{GHz} \mathrm{CPU}$ and $7.88 \mathrm{~GB}$ of memory. This section selects FaceNet algorithm [22], evolutionary community detection (ECD) algorithm [23], error accumulation sensitivity (EAS) algorithm [14] to compare with DMGBA. FaceNet algorithm is a classical clustering algorithm based-on evolution. ECD algorithm is a multi-objective evolutionary algorithm presented to detect the evolution of dynamic communities. EAS algorithm is proposed in dynamic community detection algorithm using a two-stage method to track the evolution of communities in dynamic networks. We use normalized mutual information (NMI) [24] to measure the accuracy of the algorithm's community division results, and take the community structure of the synthetic network itself as a real community structure.

4.2.2 Experimental Environment and Program. In this section, we choose SYN-FIX and SYN-VAR as experimental datasets. SYN-FIX and SYN-VAR are synthetic dynamic networks so they have community partition results. SYN-FIX consists of 128 nodes and is divided into four communities on average. The average degree of per node is 16 , nodes in other communities are connected by edges on average zout. In this paper, zout is 3 and SYN-FIX dynamical network is generated after 10 moments. Dynamic network SYN-VAR is composed of 256 nodes, the evolution of communities in this network is simulated by adding and removing nodes, forming new communities or splitting existing communities. In this paper, zout is 3 and SYN-VAR dynamical network is generated after 10 moments.

4.2.3 Experimental Results - Efficiency. Figure 2 shows the computational time of the ECD, FaceNet, LBTR, EAS, DMGBA in SYN-FIX and SYN-VAR datasets. The experimental results show that DMGBA has much better time performance than other baseline algorithms in all time steps. ECD algorithm takes the longest time. This is because DMGBA only needs to deal with incremental changes which reduces the running time of the algorithm.

4.2.4 Experimental Results - Accuracy. NMI is often used to detect the difference between the results of the division and the true partition of the network. For two different types of $A$ and $B$, the NMI is defined as follows:

$$
\text { NMI }=\frac{-2 \sum_{i=1}^{C_{A}} \sum_{j=1}^{C_{B}} C_{i j} \cdot \log \left(\frac{C_{i j} \cdot N}{C_{i} \cdot C_{j}}\right)}{\sum_{i=1}^{C_{A}} C_{i} \cdot \log \left(\frac{C_{i}}{N}\right)+\sum_{j=1}^{C_{B}} C_{i j} \cdot \log \left(\frac{C_{\cdot j}}{N}\right)}
$$

The greater the value of NMI, the more similarity between $A$ and $B$ division, when the NMI value is 1 , it indicates that $A$ and $B$ are the same as division of the network.

The experimental accuracy of DMGBA and compared methods as figure 3 shows. In SYN-FIX datasets, ECD algorithm always maintains the highest NMI with a mean of 1 , The NMI of DMGBA is lower than ECD algorithm but it also performs well with a mean of 0.9. LBTR, EAS and FaceNet have poor performance. Figure 3 also shows the NMI comparison results of these five algorithms in SYN-VAR dynamic network. It can be seen that the NMI of ECD algorithm, EAS algorithm and DMGBA shows a similar fluctuation trend. ECD algorithm has the highest NMI mean, followed by EAS algorithm and DMGBA, FaceNet algorithm has the lowest NMI mean.

Considering the efficiency of experimental results, DMGBA performs better than all comparison algorithms. Take into account the accuracy of community division results, DMGBA also has better performance. In summary, DMGBA is better than compared methods.

\section{CONCLUSION AND FUTURE WORK}

In this paper, we present MGBA to simulate the process of community evolution in static network and DMGBA to detect the community structure in dynamical network. In MGBA, we put forward 
the concept of parent node. When a vertex community is allocated to the community with the largest gravity, the central node of this community is defined as the parent node of the vertex community. Once assigned, the parent node of a community will not be changed anymore, while the central node of a community will change with the operation of the gravity model changing. Every vertex community's center node is the node itself at the beginning, then it will change as the central node of the parent node changes. MGBA only needs to investigate the neighbor nodes to adjust the hierarchical nested community structure which reduces the running time of the algorithm and get higher accuracy. On this basis of MGBA, we propose a DMGBA which is based on incremental clustering. When using MGBA to detect the community structure in static networks. If edges are added or removed, DMGBA will be used to redivide the dynamical communities. Since DMGBA is only concerned with adding edges and deleting edges, it reduces the running time of the algorithm and gets higher accuracy. A series of experiments shows that our algorithms outperform the state-of-the-art community detection methods.

In the future, we will explore the following directions:

- The nodes and edges may have weight in the networks, we will explore how to adjust the appropriate parameter of weight to further enhance the performance.

- Improving the community division which is detected by the MGBA and DMGBA.

- In this paper, using MGBA or DMGBA will get nonoverlapping community. However, in most realistic scenarios, the community are overlapping. We will try to detect overlapping community structure in social networks.

\section{REFERENCES}

[1] Coscia, M. , Giannotti, F. , \& Pedreschi, D. (2011). A classification for community discovery methods in complex networks. Statistical Analysis \& Data Mining the Asa Data Science Journal, 4(5).

[2] Newman, M. E. . (2004). Fast algorithm for detecting community structure in networks. Phys Rev E Stat Nonlin Soft Matter Phys, 69(6 Pt 2), 066133.

[3] Blondel, V. D. , Guillaume, J. L. , Lambiotte, R. , \& Lefebvre, E. . (2008). Fast unfolding of communities in large networks.

[4] Waltman, L., \& Eck, N. J. V. (2013). A smart local moving algorithm for large-scale modularity-based community detection. European Physical Journal B, 86(11), 471.

[5] Cai, X. Y. (2009). Community-finding algorithm in complex networks based on spectral clustering. Computer Science.
[6] Matthew Van Gundy, Davide Balzarotti, and Giovanni Vigna. 2007. Catch me, if you can: Evading network signatures with web-based polymorphic worms. In Proceedings of the first USENIX workshop on Offensive Technologies (WOOT 07) . USENIX Association, Berkley, CA, Article 7, 9 pages.

[7] J. Hopcroft, O. Khan, B. Kulis. (2004). Tracking evolving communities in large linked networks, Proc. Natl. Acad. Sci. USA 101(Suppl 1)5249-5253.

[8] Francesco, Folino, Clara, \& Pizzuti. (2013). An evolutionary multiobjective approach for community discovery in dynamic networks. IEEE Transactions on Knowledge \& Data Engineering.

[9] Gopal, V. E. , Prasad, M. , \& Ravi, V. (2010). A fast and elitist multiobjective genetic algorithm: NSGA-II.

[10] Scardua, L. A. . (2021). Multiobjective Evolutionary Algorithm Based on Decomposition.

[11] Jingjing, M. , Jie, L. , Wenping, M. , Maoguo, G. , \& Licheng, J. (2014). Decomposition-based multiobjective evolutionary algorithm for community detection in dynamic social networks. The Scientific World Journal, 2014, (2014-3-1), $2014,402345$.

[12] Meng, X. , Tong, Y. , Liu, X. , Shuai, Z. , Yang, X. , \& Tan, S. (2017). A novel dynamic community detection algorithm based on modularity optimization. IEEE International Conference on Software Engineering \& Service Science. IEEE.

[13] Zhao, Z., Chao, L. , Zhang, X. , Chiclana, F. , \& Viedma, E. H. (2019). An incremental method to detect communities in dynamic evolving social networks. Knowledge-Based Systems, 163(JAN.1), 404-415.

[14] Xu, Z., Rui, X. , He, J, Wang, Z. , \& Hadzibeganovic, T . (2020). Superspreaders and superblockers based community evolution tracking in dynamic social networks. Knowledge-Based Systems, 192(Mar.15), 105377.1-105377.19.

[15] Yin, G, Chi, K, Dong, Y, \& Dong, H. (2017). An approach of community evolution based on gravitational relationship refactoring in dynamic networks. Physics Letters A, 381(16), 1349-1355.

[16] Backstrom, L , Huttenlocher, D. P. , Kleinberg, J. M. , \& Lan, X. (2006). Group formation in large social networks: membership, growth, and evolution. Acm Sigkdd International Conference on Knowledge Discovery \& Data Mining. ACM.

[17] Yang, J. , \& Leskovec, J. (2012). Defining and evaluating network communities based on ground-truth. IEEE Computer Society, 1-8.

[18] Roberto González Sánchez. (2014). Measurements and analysis of online social networks. Materials Transactions Jim, 21(3), 159-168.

[19] Leskovec, J. , Kleinberg, J. , \& Faloutsos, C. (2007). Graph evolution. Acm Transactions on Knowledge Discovery from Data, 1(1), 2-es.

[20] Yang, J. , \& Leskovec, J. (2013). Community-Affiliation Graph Model for Overlapping Network Community Detection. IEEE International Conference on Data Mining. IEEE.

[21] Yang, J. , \& Leskovec, J. (2013). Overlapping community detection at scale: A nonnegative matrix factorization approach. Acm International Conference on Web Search \& Data Mining. ACM.

[22] Lin, Y. R. , Chi, Y., Zhu, S., Sundaram, H. , \& Tseng, B. L. (2008). Facetnet: A framework for analyzing communities and their evolutions in dynamic networks. Proceedings of the 17th International Conference on World Wide Web, WWW 2008, Beijing, China, April 21-25, 2008.

[23] Liu, F. , Wu, J. , Zhou, C. , \& Yang, J. (2019). Evolutionary Community Detection in Dynamic Social Networks. 2019 International Joint Conference on Neural Networks (IJCNN).

[24] Hoeschen, C. , Kontos, D. , Flohr, T. G. , Viana, R. S. , Galarreta-Valverde, M. A. , \& Mekkaoui, C. et al. (2019). Normalized Mutual Information. 